\title{
CHAPTER VI \\ REPORTS ON DIVISION, COMMISSION and WORKING GROUP MEETINGS
}

\section{DIVISION I}

PRESIDENT

VICE-PRESIDENT

PAST PRESIDENT BOARD

\author{
FUNDAMENTAL ASTRONOMY \\ (ASTRONOMIE FONDAMENTALE)
}

\author{
Jan Vondrak \\ Dennis D. McCarthy \\ Toshio Fukushima \\ Toshio Fukushima, George H. Kaplan, Joseph A. \\ Burns, Zoran Knezevic, Irina I. Kumkova, \\ Daffyd W. Evans, Aleksander Brzezinski, \\ Chopo Ma, Pascale Defraigne, Richard N. \\ Manchester, Sergei A. Klioner, Gerard Petit
}

\section{PARTICIPATING COMMISSIONS}

Commission 4

Commission 7

Commission 8

Commission 19

Commission 31

Commission 52
Ephemerides

Celestial Mechanics and Dynamical Astronomy

Astrometry

Rotation of the Earth

Time

Relativity in Fundamental Astronomy

\section{DIVISION WORKING GROUPS}

Second Realization of International Celestial Reference System

Numerical Standards of Fundamental Astronomy

Astrometry with Small Ground-based telescopes

\section{INTER DIVISION WORKING GROUPS}

Division I / Division III Cartographic Coordinates and Rotational Elements of Planets and Satellites

Division I / Division III Natural Satellites

PROCEEDINGS BUSINESS SESSIONS, 3 August and 4 August 2009

\section{Introduction}

There were four 1.5-hour sessions of Division I business meetings during the XXVIIth IAU General Assembly. The first three were devoted to the reports of Commissions, Working Groups and services associated with the Division, discussion about plans for the next triennium and future structure of the Division. Scientific presentations on the future space astrometric mission Gaia were made at the fourth session. 


\section{Business Meeting, Monday 3 August 2009, 11:00 hr}

This session, chaired by Dennis McCarthy, was devoted to the reports of all Commissions pertaining to Division I on activities 2006-2009 and plans for 2009-2012.

The report of Commission 4 (Ephemerides) was presented by its President, Toshio Fukushima.

The report of Commission 7 (Celestial Mechanics) was presented by its President, Joseph Burns. He gave a summary of the activities of the Commission during the last 3 years and of the current state of the Commission, including a list of recently deceased members, introduced the incoming officers, and emphasized the following issues:

- organization of IAU Symposium 236: Near Earth Objects, our Celestial Neighbors: Opportunity and Risk, in Prague (Czech Republic), in 2006;

- organization of IAU Symposium 249: Exoplanets, Detection, Formation and Dynamics, in Suzhou (China), in 2007;

- preparation of the Triennial Report.

The journal Celestial Mechanics and Dynamical Astronomy is in a good state. Commission 7 also spent some time considering whether, and what, symposia the commission might wish to organize. The most specific of these was a proposal to organize a 2012 symposium in Finland on the occasion of the 100th anniversary of the publication of Karl Sundman's pivotal work on regularization methods in the three-body problem. This will be considered further. Improvements to the Commission's web page were also suggested, and many have already been implemented.

The report of Commission 8 (Astrometry) was presented by its President, Irina Kumkova. She touched the following topics in which the Commission was active:

Instrumentation and Reduction Methods: The activities in Brazil (CCD heliometer), China (NEOST - Near Earth Objects Space Telescope and LAMOST - Large Sky Area Multi-object Fiber Spectroscope Telescope), France (the reduction of mosaic CCD images obtained at the $3.6 \mathrm{~m}$ CFHT in order to derive coordinates of pulsars and QSOs in the optical at the mas level), Russia (modernized automatic telescope MTM-500M for Solar System bodies astrometric and photometric observations), Ukraine (artificial satellites and space debris, small solar system bodies), UK (new reduction of the Hipparcos data) and USA (StarScan plate measuring machine completed at USNO, measuring all applicable Black Birch, AGK2, and Hamburg Zone astrograph plates) were reported.

Space Astrometry: The European Space Agency's astrometry mission Gaia is very important because of its ability to obtain accurate astrometric measurements for very large samples of stellar, extragalactic and solar-system objects, and the matching collection of synoptic, multi-epoch photometric and radial-velocity data as well as to address an extremely broad range of topics in galactic and stellar astrophysics, solar-system astronomy, reference frame and fundamental physics. JASMINE and Nano-JASMINE projects being developed in Japan. HST FGS data were used for mas-precision of astrophysically interesting stars, to establish perturbation orbits due to planetary-mass companions of nearby stars. The observations continue for RR Lyr and Pop II Cepheid stars to calibrate a Pop II Period-Luminosity relationship.

Reference Frames: Investigations and studies are active in Brazil (work on the extragalactic frame concentrates on the reconciliation between optical and radio positions). A list of 173 candidate stable sources was proposed; the precession and equinox motion correction were obtained from various samples of PPM and ACRS proper motion data in China, the Large Quasar Astrometric Catalogue was derived in France; relative positions of reference stars around ICRF radio sources were improved in Rumania.

Positions and Proper Motions: Extensive studies were made in Brazil, France, Germany, Italy, Japan, Russia, Spain, Ukraine, USA and China.

Open and Globular Clusters were studied in Brazil, China, Italy, Ukraine and USA.

Education in Astrometry: The first Chinese-French Spring School on Astrometry 'Observational campaign of solar system bodies', was held in Beijing, April 7-13, 2008. A new web-page 'Infrared Astrometry' (Kharin and Vedenicheva) was launched at the web-site of the MAO NAS of Ukraine http://www.mao.kiev.ua/IR. New trends of research oriented towards perspective usage of plate archives of MAO NAS of Ukraine as an element of Virtual Observatory was designed. A database of CCD, photographic observations and astrometric catalogues of NAO, Ukraine was made (Protsyuk) within the framework of IVOA. The databank of photographic observations of NAO is included in the WFPDB (Bulgaria). 
Symposia, Colloquia, Conferences:

- Journées 2007 The Celestial Reference Frame for the Future, Meudon, France, September 1719, 2007

- IAU Symposium 248 A Giant Step: From Milli- To Micro-Arcsecond Astrometry, Shanghai, China, October 15-19, 2007

- ADELA 2008 - IV Meeting on Dynamical Astronomy in Latin-America, Mexico City, February $12-16,2008$.

The report of Commission 19 (Rotation of the Earth) was presented by its President, Aleksander Brzeziński. Members of Commission 19 concentrated on different problems concerning Earth rotation. This phenomenon is conventionally described by the Earth Orientation Parameters (EOP) which are determined by the space geodetic techniques with continuously improving accuracy and temporal resolution. New observation techniques have been recently developed, such as the ring laser gyroscope and the new generation system VLBI2010 with small antennas. The advances in monitoring variations in Earth rotation are closely connected to the establishment of the IAG Global Geodetic Observing System (GGOS). Besides tidal influences of the external bodies, the principal causes of EOP variation are changing motions and mass distribution of the fluid parts of the planet. Therefore the development of observations and modeling of geophysical fluids, the atmosphere, oceans, and land hydrosphere, has been considered as an important mean for understanding Earth rotation variability.

Considerable progress could be achieved thanks to the observations of mass redistribution done by the gravity recovery and climate satellite experiment GRACE. Commission 19 closely cooperated with the International Earth Rotation and Reference Systems Service (IERS) on updating the IERS Conventions which provide a set of astronomical and geodetic constants and fundamental procedures. Besides determination of the EOP and the related geophysical excitation parameters, there were also many efforts to improve forecasts of these quantities, which are needed for spacecraft navigation and other practical purposes. Further refinement of the theoretical description of Earth rotation is considered as an important task for the commission activity during the next term. It is planned to organize for this purpose a common IAU/IAG Working Group on 'Theory of Earth rotation'.

President of Commission 31 (Time), P. Defraigne, presented the main results achieved by the commission members during the last triennium. Concerning the relative frequency stability and accuracy of the atomic time scales, the 1-month stability of the International Atomic Time (TAI) is currently close to $3 \times 10^{-16}$, which is a limit of present clock performance. However, the long term stability will be improved in the near future thanks to improvements of the algorithms. The last version of TT(BIPM) was also presented with its present uncertainty of $5 \times 10^{-16}$. The recommendations of the Consultative Committee for Time and Frequency having an impact on the Astronomical Activities have also been reported. The pulsar time scales were shown to achieve a stability of $10^{-15}$ at 7 years.

The meetings relating to the scope of the Commission that were held during the triennium, in addition to the usual meetings of the time and frequency community, comprised the Fifth International Time Scale Algorithm (San Fernando, April 2008), IAU Symposium 261 'Relativity in Fundamental Astronomy: Dynamics, Reference Frames, and Data Analysis', (Virginia Beach, April 2009) and the Joint Discussion 'Time and Astronomy' during the 27th IAU General Assembly in Rio.

The activities of Commission 52 (Relativity in Fundamental Astronomy) were presented by its President, Sergei Klioner. The general goals of this newest Commission, created in 2006, are to clarify geometrical and dynamical concepts of Fundamental Astronomy within a relativistic framework; to provide adequate mathematical and physical formulations to be used in Fundamental Astronomy; to deepen the understanding of the obtained results among astronomers and students of astronomy, and to promote research needed to accomplish these tasks.

The projects on which the Commission works are the following:

- Compile a list of unresolved problems;

- Frequently asked questions;

- Relativistic glossary for astronomers;

- 'Task teams', i.e. ad hoc discussion groups for well posed issues (e.g., for TDB units). 
The Commission understands the 'Applied Relativity' as a multidisciplinary research topic, with important social and educational problems:

a) People doing practical work have limited knowledge of relativity and often can not understand the details of the suggested relativistic models.

b) People working in relativity have limited experience with real data and often can not judge if what they suggest is at all relevant.

So, basic education in relativity should be a part of astronomical education and discussions between the two above mentioned groups are necessary.

The above mentioned problems were discussed at IAU Symposium 261 'Relativity in Fundamental Astronomy: Dynamics, Reference Frames, and Data Analysis' (Virginia Beach, USA, 27 April - 1 May, 2009), with 92 participants from 18 countries.

The topics on which the work will be mainly concentrated in the future are the definition of the ecliptic (or 'ecliptic image') in the Geocentric Celestial Reference System (GCRS), and the system of astronomical units.

\section{Business Meeting, Monday 3 August 2009, 14:00 hr}

This session, chaired by Jan Vondrák, was devoted to the reports of Division I Working Groups and associated Services.

WG Second Realization of the International Celestial Reference Frame (ICRF2), led by Chopo Ma, prepared the solution that is based on VLBI data in S/X bands through March 2009. 4540 sessions were used with about 6.5 million observations of 3414 different radio sources. Out of these, 295 sources are defining, position uncertainty of individual sources is $\geqslant 40 \mu \mathrm{as}$, the accuracy of the orientation of the axes is $10 \mu \mathrm{as}$. The orientation is independent of the equator, ecliptic and equinox. The solution was accepted by the WG for ICRF2, and the Directing Boards of the IERS and IVS. All details of the solution are given in IERS Technical Note 35 'The second realization of the International Celestial Reference Frame by Very Long Baseline Interferometry', published by Bundesamt für Kartografie und Geodäsie, Frankfurt a.M., Germany, and is also available in PDF format at http://iers.org/MainDisp.csl?pid=46-25772. The WG proposed the IAU resolution on ICRF2, and if it is approved by the IAU General Assembly, the WG is to be disbanded.

Brian Luzum presented the report on the WG Numerical Standards of Fundamental Astronomy (NSFA) that he chairs. The WG was established at the IAU 2006 General Assembly to update the IAU Current Best Estimates (CBE) of astronomical constants while conforming with existing resolutions and conventions where possible. In order to ensure consistency, the Working Group coordinated with IAU Commissions 4 and 52, the IERS, and the BIPM Consultative Committee for Units. Following extensive discussions by e-mail as well as a few meetings, a new set of CBE was proposed for use as the IAU 2009 System of Astronomical Constants, and the corresponding IAU resolution was prepared, to be approved by the IAU General Assembly. In the 2009-2012 triennium, the WG will establish the procedures for a CBE electronic document and resolve the differences regarding the future of the astronomical unit. It is anticipated that IAU Division I will, in the 2009-2012 triennium, find a permanent home for the responsibilities of the WG.

Report on the activities of the WG Astrometry by Small Ground-based Telescopes was presented by William Thuillot, chairman of the WG, which includes 29 people from 12 countries. The goal of the WG is to maintain information on the astrometric activities performed with telescopes having diameters up to 2 meters, to facilitate the collaboration between the teams, and to encourage coordinated activities. These instruments are numerous, worldwide, generally easy to access and are therefore well adapted to medium and long-term programs and to observations in networks. They are well adapted to the teaching of practical astronomy and astrometry. Thus the WG is also interested in teaching astrometry. A web site located at www.imcce.fr/astrom/ disseminates information on the WG activity. Several research teams are involved in the WG, several groups are already collaborating and all are very active in the WG activity domain.

The following activities are performed: Zacharias et al. in USA, densification of reference frames; Van Altena et al., stellar astrometry and the providing of the Yale/San Juan Southern Proper Motion Catalog; Muinos et al. in Spain, stellar astrometry and the providing of the Carlsberg Meridian Catalog; Wenjing et al. in China, asteroids astrometry and natural satellites 
studies; Vieira-Martins, Assafin et al. in Rio, natural satellites astrometry and optical counterpart of extragalactic sources useful for the celestial reference frame; Pinigin et al. in Ukraine collaboration with teams in Kazan, Istanbul and Shanghai, space debris and Near-Earth asteroids; Pauwels et al. in Belgium, asteroids astrometry; Souchay et al. in Paris, astrometry of extragalactic sources and GBOT project of astrometry of the Gaia probe; Thuillot, Arlot et al. in Paris, mutual events of natural satellites campaigns and a ground-based follow-up network for the Solar System objects detected by Gaia.

Two specific actions were organized by members of the Working Group:

- In order to gather information on astrometry teaching, a census has been performed in March 2008 and several interesting answers were received (see the WG website).

- A spring school of astrometry was organized and held on 7-12 April 2008 in Beijing, China, with the topic 'Observational campaigns for Solar System bodies'. $28 \mathrm{PhD}$ and postdoc students attended this training.

The report of WG Cartographic Coordinates and Rotational Elements was presented by its chairman, Brent Archinal. The WG, now consisting of 18 members from 10 countries, was established in 1976, and belongs to Divisions I and III. So far it prepared and published nine reports. Certain concerns were expressed about possible 6-year limits on WG chairs and limits on WG existence. The WG is now finishing the discussion on new issues for the triennial report. The present status of the current WG progress/issues is the following:

Mercury model: New radar results (J. Margo); continued discussions about feature-based vs. axis-of-figure system.

Moon: DE 421 ephemeris and mean Earth/polar axis system (recommendation from NASA Lunar Geodesy and Cartography WG).

Mars: No changes (recommendation from NASA Mars Geodesy and Cartography WG).

Saturnian moons: New results from Cassini; Titan, icy Satellites, Saturn rotation.

Jupiter/Saturn/Neptune rotation? Expertise needed - help wanted!

Small bodies: Subgroup to decide which to include.

Category for dwarf planets: Separate category for now; will possibly treat same as small bodies. Long term issues (not resolved now): Work on re-establishing International Association of Geodesy affiliation; develop recommendations for extra solar planets.

Jean-Eudes Arlot reported on WG Natural Satellites that he is chairing. It belongs to Divisions I and III because of its interest both in dynamics and physics. The working group coordinates the study of the families of satellites:

- main satellites such as Galileans, eight Saturnians, five Uranians, Triton. They are small solar systems interesting because of resonances, Laplacian libration of the Galileans, evolution of the systems, internal dissipation of energy.

- outer irregular satellites: formation and evolution

- inner satellites: relationship with rings, evolution.

Some of these objects are goals for the space probes: Jupiter-Europa: project 'Laplace'; SaturnTitan: project 'Tandem'; Pluto-Charon: mission 'New Horizons'. The topics of interest of the working group are: Celestial mechanics, Astrometry; Physics of the surfaces and internal structures; Formation and evolution of the solar system; Astrometry of the giant planets reachable through their satellites. The studies of the working group are of interest for the planets: the giant planets are difficult to observe for astrometric purpose, but the satellites are easier to observe and their ephemerides provide the position of the center of mass of the planet.

Works performed during the last triennium: Observations:

- USNO (Flagstaff): transit circle observations,

- USNO/IMCCE/Brussels/Pulkovo: scan of old plates,

- CNPq Brazil: CCD observations of all systems,

- IMCCE: mutual events campaigns (Uranus, Saturn, Jupiter),

- MPC: gathering outer satellites observations.

Ephemerides:

- JPL: all satellites (R.A. Jacobson),

- IMCCE and SAI: all satellites (J.E. Arlot, N. Emelianov),

- IAA: Galilean satellites (G. Krasinsky),

- MPC: outer irregular satellites,

- IMCCE and SAI: extrapolation of errors in ephemerides.

Note that observations are needed each year for all satellites! Some faint outer satellites were 
observed only once by large telescopes. So, they are lost soon. The observations have been stopped after 2003 or 2006, depending on the satellites.

The astrometric database NSDC is maintained through the working group. The main interest of the working group is to gather all available astrometric observations and to encourage the observational campaigns needed to be international, i.e. organized worldwide. The members of the working group help keep the database exhaustive (at 90\%). It is maintained thanks to IMCCE (Paris) and SAI (Moscow).

The WG encourages the improvement of astrometric accuracy by means of: - evaluation of the distance center of mass photocenter (need to know the surfaces);

- taking into account the changing absorption of the sky for moving objects (need monitoring of a photometric source);

- the star catalogue used for the reduction (corrections of zonal errors, use of a new catalogue UCAC3, GAIA,);

- re-reducing the scanned old photographic plates with new methods and new star catalogues.

The activities of the International Earth Rotation and Reference Systems Service (IERS) were described by the chairman of its Directing Board, Chopo Ma. The news from the IERS Product Centers is the following:

- Earth Orientation: Web service for Earth Orientation Parameters (EOP) and Earth orientation matrix; new EOP series IERS05 C04 consistent with the new terrestrial system ITRF2005 (see below); revision of Bulletin B, following community survey.

- Rapid Service / Prediction: Bulletin A changed to match IERS05 C04; initiated WG on prediction; web site moved and revised considerably.

- International Terrestrial Reference System (ITRS): Released ITRF2005, based on time series of SINEX files, containing site positions and EOP as combined by the Technique Centers, where available; issued call for participation for ITRF2008, now in progress; participation in some local surveys of co-located sites.

- International Celestial Reference System (ICRS): Increased VLBI observations in the southern hemisphere; edited IERS Technical Note 35 on ICRF2; drafted resolution on ICRF2 for IAU GA.

- IERS Conventions: Updated various chapters in online version IERS Conventions (2003); preparing for new Conventions (2009).

- Global Geophysical Fluids Center: There are eight Special Bureaus (Atmosphere, Core, Gravity/geocenter, Hydrology, Loading, Mantle, Oceans, Tides) that were reviewed by the IERS Directing Board and will be re-constituted. A call for proposals was released in May 2009, with the goal to establish operational products and include new products.

Combination Research Centers are to be replaced by Research Centers, responsible for specific topics related to Product Centers. Working Groups are established for:

- Prediction (studying EOP prediction at various time scales);

- Site Survey and Co-location (reconstituted with the new charter, chair and membership);

- Combination (will be ended on completion of Combination Pilot Project);

- Combination at the Observation Level (newly established);

- ICRF2.

The Central Bureau in Frankfurt further developed the IERS Data and Information System (DIS), based on modern technologies for internet-based exchange of data and information using XML and the generation and administration of ISO metadata. It also provides access to and visualization of IERS data products, and links to IERS components.

There were several workshops organized:

- Workshop on Global geophysical Fluids (San Francisco, CA, USA, December 6-7, 2006);

- Workshop on Conventions (Sèvres, France, September 20-21, 2007);

- FFOS Unified Analysis Workshop (Monterey, CA, USA, December 5-7, 2007);

- GGOS Unified Analysis Workshop, Follow-Up Meeting (Vienna, Austria, April 15, 2008), and several IERS publications produced (IERS Technical Notes 34 and 35, IERS Annual reports 2005 and 2006, IERS Bulletins A, B, C and D - weekly to semi-annually, IERS Messages No. 92 trough 149).

The work done at the International VLBI Service for Geodesy and Astrometry (IVS) was described by Harald Schuh. During the report period the IVS held two General Meetings, 
one in Concepción, Chile in January 2006 and the other in Saint Petersburg, Russia in March 2008. Further, two Technical Operations Workshops were held at MIT Haystack Observatory in Westford, MA in April/May 2007 and April 2009, respectively. Another important meeting was the VLBI2010 Workshop on Future Radio Frequencies and Feeds (FRFF) in Wettzell, Germany in March 2009. The FRFF workshop resulted in recommendations for the VLBI2010 frequency range and a demonstration campaign in 2012, which were subsequently endorsed by the IVS Directing Board.

The IVS completed its first ten years of being a service for geodetic and astrometric VLBI on March 1, 2009. To commemorate the first decade a 10th Anniversary Celebration event was held in Bordeaux, France on March 25, 2009. The event included a symposium featuring the history of VLBI and the IVS, the interrelation of the IVS with the other space geodetic services (IGS, ILRS, IDS), and IVS' place among the other VLBI networks (EVN, VLBA, Asian networks). The event was live broadcast over the Internet. A recording of the various presentations is available at http://canalc2.u-strasbg.fr/video.asp?idvideo=855.

In August 2008, a 15-day continuous VLBI observation campaign called CONT08 was observed. The network consisted of eleven IVS stations. Unlike the CONT05 campaign, CONT08 was observed on the basis of UT days, i.e., an observing day was run from 0 UT to 24 UT. Observational gaps between the single observation days (30 min in the CONT05 case) were avoided by performing the daily station checks (e.g., pointing) not at the change of schedules but at well-coordinated, staggered times for all stations (i.e., different daily check times for each station). A special issue of Journal of Geodesy on CONT08 is in the planning stage.

The IVS VLBI2010 Committee (V2C) submitted a Progress Report on the status of the development of the next generation geodetic VLBI system (VLBI2010 system), which summarizes the progress made in the development of the new system up to the end of 2008 . The report was published as a NASA Technical Memorandum TM-2009-214180 and is available online on the IVS Web site.

The report on Standards of Fundamental Astronomy (SOFA) was presented by Patrick Wallace. SOFA is a service operated by Division I to provide authoritative fundamental-astronomy algorithms, for example, precession-nutation models. The activity is carried out by an international board that includes both astronomers and software experts, and has cross-membership with the IERS Directing Board.

During the period 2006-2009, the SOFA software collection has grown from 121 routines to 160 , the increase being due to the introduction of the IAU 2006 precession. The software has a core of 41 canonical models, used by 67 astronomy-related routines and supported by 52 vector-matrix utilities.

The triennium saw the development of a second version of the SOFA software, this time in the $\mathrm{C}$ programming language and supported in parallel with the existing Fortran version. At the same time, the SOFA licensing conditions have been made more liberal, to harmonize with IAU/IERS practices in general and to encourage the widest possible use. SOFA's products are disseminated through a website: http://www.iau-sofa.rl.ac.uk/.

Considerable thought has been given to SOFA's formal position within the IAU organization, with the object of raising its profile and increasing user confidence while at the same time complying with IAU statutes, bye-laws and working rules. These discussions are still in progress.

\section{Business Meeting, Monday 3 August 2009, 16:00 hr}

The session was devoted to general problems of the Division and discussion on its future structure, and was chaired by Sergei Klioner and Dennis McCarthy.

Nicole Capitaine, IAU Representative to the Comité Consultatif des Unités (CCU), reported on the topics discussed during the recent CCU meeting 2009, relevant to Division I.

1) The CCU was consulted on the proposal by Capitaine \& Guinot (2008) to the IAU NSFA Working Group about the astronomical unit (ua) of length. According to that proposal, the ua would be re-defined trough a fixed relation to the SI meter by a defining number. The ua would then no more be determined experimentally, which would directly permit the possible variation of the mass of the Sun, and/or of $G$. This would limit the role of the ua to that of a unit of length of 'convenient' size for solar system applications. The CCU declared its support to move to a fixed relationship to the SI meter through a defining number determined by continuity with past determinations. The CCU, also consulted on the symbol to be used ('ua' or 'au'), suggested 
that the astronomers should decide upon an appropriate symbol for this unit independently of the language.

2) The CCU discussed a Note by Capitaine \& Guinot suggesting improvements in the definition of the second, namely (i) that the definition should distinguish the concept and its realization and (ii) that the SI brochure make clear that the SI units ( $\mathrm{s}$ and $\mathrm{m}$ ) should be used for coordinate quantities in GR (e.g. the second for the coordinate times TDB and TT). This is in agreement with the conclusion of a paper by Klioner et al. (2009) within IAU Commission 52 on Relativity in Fundamental astronomy. This was acknowledged by the CCU.

3) Following a problem raised by the International Union of Pure and Applied Chemistry (IUPAC) about the fact that there exists no official definition of the year, and that the symbols 'yr', 'y' and 'a' are all used, the CCU established a small group in order to propose a formulation for the definition of the year, and an official symbol. That group would include a representation from IAU (N. Capitaine), IUPAP and ICRU (International Commission on Radiation Units and Measurements) and should report at the next CCU meeting.

Jan Vondrák then reported on the activities of Division I (Fundamental Astronomy). He reminded the audience of the present structure (6 Commissions, 5 Working Groups, including the two common with Division III, and 3 associated Services), and stressed that most of the scientific activities are made through individual Commissions and Working Groups that reported during the first two sessions. In the preceding triennium, Division I coordinated and/or supported the following IAU meetings:

- IAU Symposium 248 A Giant Step: from Milli- to Microsecond Astrometry, October 15-19, 2007, Shanghai, China;

- IAU Symposium 249: Exoplanets, Detection, Formation and Dynamics, October 22-26, 2007, Suzhou, China;

- IAU Symposium 261 Relativity in Fundamental Astronomy, April 27 - May 1, 2009, Virginia Beach, USA;

- IAU Joint Discussion 6 Time in Astronomy, August 5-6, 2009, Rio de Janeiro, Brazil;

- IAU Special Session 9 Marking the 400th Anniversary of Kepler's Astronomia Nova, August 11-14, 2009, Rio de Janeiro, Brazil;

- Mathematics and Astronomy, a Joint Long Journey, November 23-27, Madrid, Spain.

Organizational activities of the Division President consisted mainly in regular annual evaluation of proposals for IAU Symposia (early 2007 - Symposia in 2008; May 2008, common meeting of Division Presidents with IAU Executive Committee in Oslo - Symposia, Joint Discussions, Special Sessions at the 27th IAU General Assembly in 2009; early 2009 - Symposia in 2010). The recommendations of DP were mostly accepted by IAU EC, with only a few exceptions.

Elections of the new President (P), Vice-President (VP) and Organizing Committee (OC) for the next triennium 2009-2012. The former VP, Dennis McCarthy agreed to serve as P for the next period. Next VP was elected by all members of the Division I (about 700) in e-mail ballot from the two candidates (Aleksander Brzeziński and Sergei Klioner), proposed by the outgoing OC. Sergei Klioner received a majority of votes and becomes the next VP. For the selection of the next OC membership the Division followed the same practice as introduced at the 26th IAU GA in Prague: OC consists of Presidents and Vice-Presidents of all six Commissions pertaining to Division I.

During the last year or so, an electronic discussion on the future structure of the Division took place, among OC members and some members of WG's. The main goal was to find a more permanent place for the people taking care of the astronomical constants, numerical standards, software etc... than a Working Group. There were several possibilities open, e.g., to create a new Commission, or to widen the scope of an existing Commission (C4?). However, none of the proposed solutions was acceptable for all concerned, and no consensus was found. Therefore it was decided that a discussion will be organized at this meeting with a goal to find a solution for the future.

The following discussion was led by Dennis McCarthy. He began by outlining the potential issues for the future:

- Astronomical constants 
- Gaussian gravitational constant, Astronomical Unit, GMSun, geodesic precession-nutation

- Astronomical software?

- Solar System Ephemerides?

- Pulsar research

- Comparison of dynamical reference frames

- Future Optical Reference Frame

- Future Radio Reference Frame

- Exoplanets: Detection, Dynamics - Predictions of Earth orientation

- Pulsar timing

- "Units of measurements" for astronomical quantities in relativistic context

- "Astronomical units" in the relativistic framework

- Time-dependent ecliptic in the GCRS - Asteroid masses

- Review of space missions

- VLBI on the Moon

- Detection of gravitational waves

After the discussion it was decided that an ad hoc sub-committee will be formed to prepare the proposals for the changes in Division I structure, to be adopted at the next IAU GA.

\section{Scientific Meeting on Gaia, Tuesday 4 August 2009, 11:00 hr}

The session was chaired by Jan Vondrák; four scientific papers, describing the ESA project Gaia from different points of view, were presented.

Gaia project: current status from ESA perspective was presented by Jos de Bruijne, working in the Gaia Project Scientist Support Team of the European Space Agency. He presented the status of the Gaia spacecraft development. The main message was that Gaia is moving from the paper and design to the hardware and testing phase. The torus (optical bench) has been brazed successfully, the various mirrors are being polished, and the CCD production is close to finalization. The next major milestone is the spacecraft Critical Design Review (CDR), which is due mid 2010. With a launch foreseen in 2012, the final Catalogue is expected in the 2020-2021 timeframe.

Dimitri Pourbaix presented the paper Gaia astrometry: accuracy and processing for different classes of objects, in which he described in detail how Gaia will scan the sky, the expected standard errors of the positions in terms of the magnitudes (for single stars ranging from magnitude 6 to 20 from 6 to $200 \mu$ as), and how non-single stars will be treated. An important part of the presentation was devoted to exoplanet detection, and also to the Solar System Astrometry (orbits, masses from close encounters, diameters, photometric data, testing of General Relativity from perihelion precessions etc...).

The paper Gaia photometry and spectroscopy: accuracy, data processing and applications was presented by Laurent Eyer. He described the payload of the satellite, and concentrated on G-band photometry with Sky Mapper and Astrometric Field. The expected precision decreases with the growing magnitude, roughly from 1 mmag to 20 mmag for magnitudes between 10 and 20. It is estimated that between 50 and 150 million variable objects will be detected. Low resolution spectro-photometry with Blue and Red Photometer (BP, RP) CCDs will be made with the goals to determine (i) color to be used for the chromatic astrometric corrections, and (ii) astrophysical parameters of the observed sources; about one billion stars will be observed from magnitude 6 to 20. Radial Velocity Spectrometer (RVS) CCDs will be used to determine radial velocities of about 150 million stars up to magnitude 17.

Sergei Klioner then concluded the session with his talk Astrophysical applications of Gaia astrometry, where he touched on many astrophysical applications that can be expected from the Gaia mission. Gaia, being an astrometric project, is expected to have a deep impact on many areas of astronomy and astrophysics. This impact comes from the extremely powerful combination of three distinct qualities in a single instrument:

- very accurate, global and absolute astrometric measurements,

- large and complete (flux limited) sample of objects,

- the matching collection of multi-epoch spectro-photometric and radial-velocity measurements. Giving a short overview of the expected results, one can mention (1) galactic structure and dynamics (large, volume-complete samples allow the determination of spatially resolved statistics in phase space); (2) stellar astrophysics (more than a million stars with distances known at about $1 \%$, calibration of the standard candles, etc.); (3) reference frame with more than 1500 object per square degree, direct link to the quasars, final accuracy of about $0.4 \mu a s / y r ; ~(4)$ fundamental physics (light deflection to about $10^{-6}$; low frequency gravitational waves; etc.). 